\title{
Nanohelices as Motion Converters
}

\author{
Bradley E. Kratochvil*, Lixin Dong, Li Zhang, Jake J. Abbott, and Bradley J. Nelson
}

\begin{abstract}
Few rotational actuators currently exist with the ability to transmit motion at different speeds, torques, and directions at the nanometer scale. We present work regarding the application of helical nanobelts as linear-to-rotary and rotary-to-linear motion converters. We discuss their ability to rectify device rotation to linear motion for untethered microrobotic applications as well as their application as rotary sample stages for nanoscale imaging.
\end{abstract}

\section{INTRODUCTION}

Previous attempts at building nanoscale rotational actuators have often focused on bearings [1], geared mechanisms, or molecular motors [2]. We present a new type of solid-state actuator capable of converting between linear and rotational motion based on nanohelices [3].

Helical nanobelts have the capacity to convert rotating magnetic fields to translational motion in a low Reynolds number regime, which provides the first step toward artificial bacterial flagella for untethered swimming microrobots [4]. By engineering nanohelicies with dual-chirality, they can be utilized as solid-state linear-to-rotation converters with a high conversion ratio between linear and rotational motion. Their compact size allows in situ deployment for applications such as three-dimensional microscopy and nanorobotic manipulation.

\section{ROTARY-TO-LINEAR}

A number of robotic swimming methods have been proposed at relatively small scales. These methods often rely on reciprocating motions, which will suffer from scaling effects as they are reduced in size. Another approach is to take inspiration from biological systems and fabricate helical propellers that mimic bacterial flagella. These devices show promise in wireless biomedical applications [5].

The helical swimming robot consists of two parts: a helical body and a soft-magnetic metal head stacked by $180-200 \mathrm{~nm}$ thick $\mathrm{Cr} / \mathrm{Ni} / \mathrm{Au}$ thin films. The helical body is fabricated in $2 \mathrm{D}$ as a InGaAs/GaAs/Cr trilayer nanoribbon and then the metal head is prepared by lift-off process (Fig. 1). The 2D patterned films are detached from the GaAs wafer and self-organized to form a one-end-fixed robot. In the experiment, the InGaAs/GaAs/Cr trilayer has thickness of $42 \mathrm{~nm}$, the ribbon width is $1.5 \mu \mathrm{m}$, and the diameter of the as-fabricated nanohelix is approximately $4 \mu \mathrm{m}$. The rectangular head has varied size from $2 \mu \mathrm{m}$ to $4 \mu \mathrm{m}$ (Fig. 2).

All authors are with the Institute of Robotics and Intelligent Systems, ETH Zurich, Switzerland.

* Corresponding Author: Bradley Kratochvil is with the Institute of Robotics and Intelligent Systems, Tannenstrasse 3, Zurich, Switzerland. (tel: +41 44632 6921; fax: +41 4463210 78; e-mail: bkratochvil@ethz.ch)

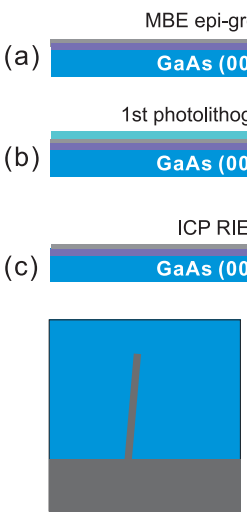

(g)
E epi-growth

01)

\section{(a)}$$
\text { 1) }
$$

IE

01)

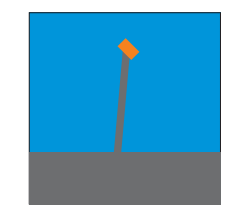

(h) (d)

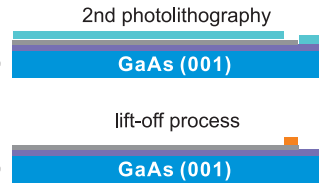

(e)
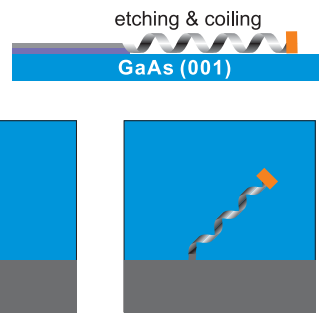

(i)
Fig. 1. Fabrication flow of the helical propeller: (a) MBE epitaxial growth of the AlGaAs sacirificial layer and InGaAs/GaAs bilayer on GaAs (001) wafer. (b) Cr layer evaporation and 1st lithography using positive photoresist $\mathrm{S} 1818$ as a mask. (c) $\mathrm{InGaAs} / \mathrm{GaAs} / \mathrm{Cr}$ trilayer is patterned to ribbon-like shape by ICP RIE using gases mixture of $\mathrm{Cl} 2$ and $\mathrm{Ar}$. (d) 2nd lithography using negative photoresist AZ 5214E as a mask. (e) e-beam evaporation of $\mathrm{Cr} / \mathrm{Ni} / \mathrm{Au}$ metal thin films and then lift-off process. (f) Wet etching of the AlGaAs sacrificial layer to release the patterned $\mathrm{InGaAs} / \mathrm{GaAs} / \mathrm{Cr}$ trilayer and the metal pad from the substrate. The InGaAs/GaAs/Cr ribbon will coil up and form a nanohelix. (g-i) top view of fabrication steps of (c), (e), and (f) respectively.

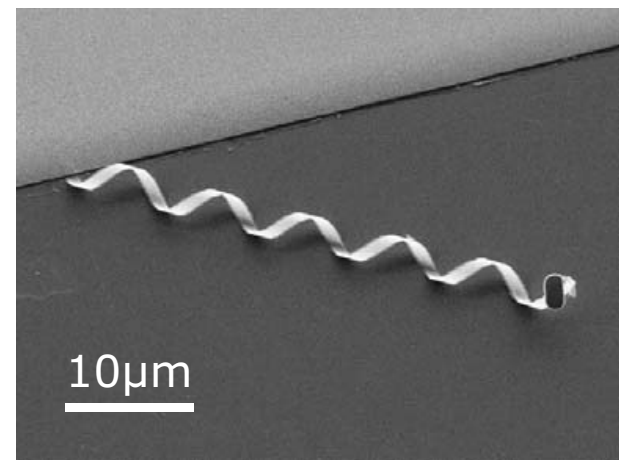

Fig. 2. SEM image of the InGaAs/GaAs swimming robot.

By adjusting the rotating speed and direction of the magnetic field, velocity and direction of motion of the helical swimmer can be tuned in a controlled fashion. Fig. 3 shows an example whare a $74 \mu \mathrm{m}$ long helical swimmer is driven to reach a target. The the average velocity is approximately $5.0 \mu \mathrm{m} / \mathrm{s}$ at $470 \mathrm{rpm}$. By inversing the rotating magnetic field, the swimmer turns in the opposite direction, and the linear motion is reversed. 


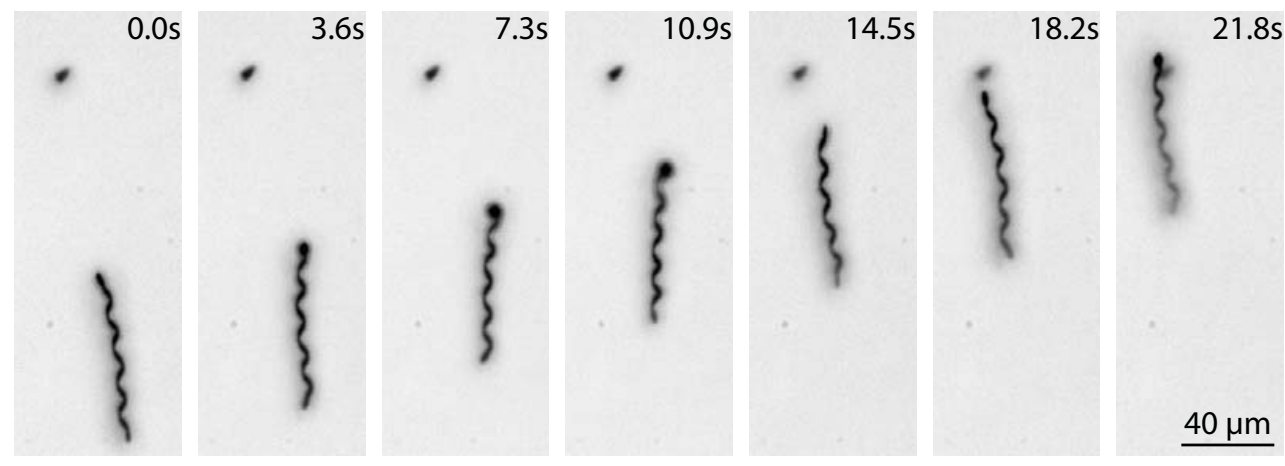

Fig. 3. time-lapse images of helical swimmer motion

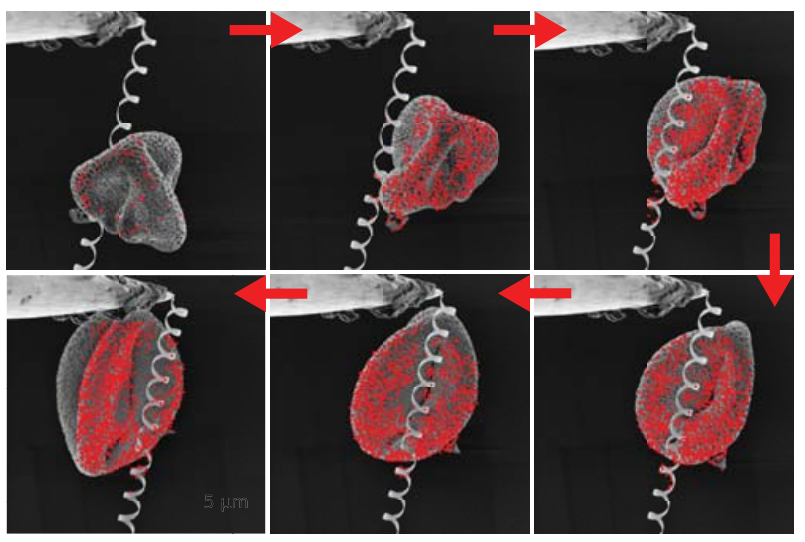

(a) frames of rotation sequence

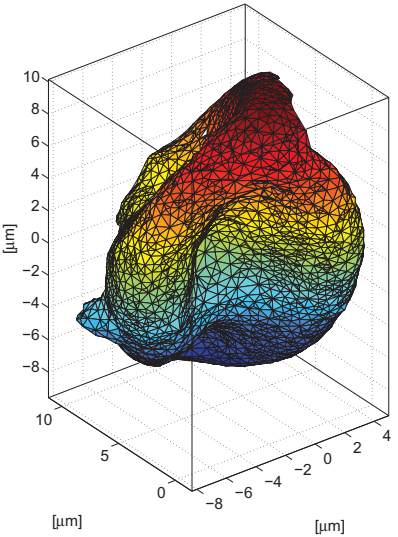

(b) meshed 3D point cloud

Fig. 4. SEM image series of a pollen grain being rotated by a nanohelix.

\section{LINEAR-TO-ROTARY}

Fundamental to the task of generating three-dimensional information from a sample in an electron microscope is the ability to view the sample from a variety of different angles. Previously, the inability to generate local rotations at suitable scales has required any rotational actuator to be accompanied by translational actuators that move the area of interest to the estimated rotation axis. Due to factors such as calibration, drift, and backlash, the system does not perform a true eucentric rotation and additional steps must be taken for data acquisition.To avoid these problems, a dual-chirality nanobelt can be used to generate localized rotations, thus simplifying the requirements of the sample stage. The use of localized goniometers allows for a simpler sampling paradigm, an increased specimen rotation range, and reduces the requirements for the automated translation stage and the data acquisition.

Figure 4(a) shows an experiment where an individual pollen grain is attached to a nanohelix and rotated. For converting linear-to-rotary motion, the nanobelt must reverse coiling directions somewhere along its body. By extending the nanohelix, a rotation is induced at the point where coiling direction changes. This mechanism has been reported to provide a $171.3^{\circ}$ rotation for a $1 \mu \mathrm{m}$ translational displacement [3], which makes it a highly efficient solid-state motion converter. Using techniques such as structure-frommotion [6], the motion can be tracked and three-dimensional data can be gathered from the sample such as in Figure 4(b).

\section{REFERENCES}

[1] A. M. Fennimore, T. D. Yuzvinsky, W.-Q. Han, M. S. Fuhrer, J. Cumings, and A. Zettl, "Rotational actuators based on carbon nanotubes," Nature, vol. 424, pp. 408-410, Jul 2003.

[2] J. V. Hernández, E. R. Kay, and D. A. Leigh, "A reversible synthetic rotary molecular motor," Science, vol. 306, no. 5701, pp. 1532-1537, Nov 2004.

[3] L. X. Dong, L. Zhang, B. E. Kratochvil, K. Shou, and B. J. Nelson, "Dual-chirality helical nanobelts: A novel linear-to-rotary motion converter," in IEEE International Conference on Micro Electro Mechanical Systems, no. 21, Jan 2008.

[4] D. J. Bell, S. Leutenegger, L. X. Dong, and B. J. Nelson, "Flagellalike propulsion for microrobots using a magnetic nanocoil and rotating electromagnetic field," in Proceedings of the IEEE International Conference on Robotics and Automation, Apr 2007.

[5] J. J. Abbott, K. E. Peyer, L. X. Dong, and B. J. Nelson, "How should microrobots swim?" in International Symposium of Robotics Research, Nov 2007.

[6] J.-P. Tardif, A. Bartoli, M. Trudeau, N. Guilbert, and S. Roy, "Algorithms for batch matrix factorization with application to structurefrom-motion," in IEEE Conference on Computer Vision and Pattern Recognition, Jun 2007, pp. 1-8. 